\title{
Control of the Proinflammatory State in Cystic Fibrosis Lung Epithelial Cells by Genes from the TNF- $\alpha$ R/NF $k$ B Pathway
}

\author{
Ofer Eidelman', Meera Srivastava', Jian Zhang', Ximena Leighton', Joshua Murtie ${ }^{1}$, Catherine \\ Jozwik $^{1}$, Ken Jacobson ${ }^{3}$, Debra L. Weinstein ${ }^{2}$, Eleanor L. Metcalf ${ }^{2}$, and Harvey B. Pollard ${ }^{1}$
}

${ }^{1}$ Departments of Anatomy, Physiology, and Genetics, and Institute of Molecular Medicine F. Edward Hebert School of Medicine, Uniformed Services University of the Health Sciences, Bethesda, Maryland, USA, 20814 ${ }^{2}$ Department of Microbiology and Immunology, F. Edward Hebert School of Medicine, Uniformed Services University of the Health Sciences, Bethesda, Maryland, USA, 20814

${ }^{3}$ Molecular Recognition Section, Laboratory of Chemistry, NIDDK, NIH, Bethesda, Maryland, USA, 20892

Accepted June 4, 2001

\begin{abstract}
Background: Cystic fibrosis (CF) is the most common, lethal autosomal recessive disease affecting children in the United States and Europe. Extensive work is being performed to develop both gene and drug therapies. The principal mutation causing $\mathrm{CF}$ is in the CFTR gene ([AF508]CFTR). This mutation causes the mutant protein to traffic poorly to the plasma membrane, and degrades CFTR chloride channel activity. CPX, a candidate drug for CF, binds to mutant CFTR and corrects the trafficking deficit. CPX also activates mutant CFTR chloride channel activity. CF airways are phenotypically inundated by inflammatory signals, primarily contributed by sustained secretion of the proinflammatory cytokine interleukin 8 (IL-8) from mutant CFTR airway epithelial cells. IL-8 production is controlled by genes from the TNF- $\alpha \mathrm{R} / \mathrm{NF} \kappa \mathrm{B}$ pathway, and it is possible that the CF phenotype is due to dysfunction of genes from this pathway. In addition, because drug therapy with CPX and gene therapy with CFTR have the same common endpoint of raising the levels of CFTR, we have hypothesized that either approach should have a common genomic endpoint.

Materials and Methods: To test this hypothesis, we studied IL-8 secretion and global gene expression in IB-3 CF lung epithelial cells. The cells were treated by either gene therapy with wild-type CFTR, or by pharmacotherapy with the CFTR-surrogate drug CPX. CF cells, treated with either CFTR or CPX, were also exposed to Pseudomonas aeruginosa a common chronic pathogen in CF patients. CDNA microarrays were used to assess global gene expression under the different conditions. A novel bioinformatic algorithm (GENESAVER) was developed to identify genes whose expression paralleled secretion of IL-8.

Results: We report here that IB3 CF cells secrete massive levels of IL-8. However, both gene therapy with CFTR and drug therapy with CPX substantially suppress IL-8 secre-

tion. Nonetheless, both gene and drug therapy allow the CF cells to respond with physiologic secretion of IL-8 when the cells are exposed to $P$. aeruginosa. Thus, neither CFTR nor CPX acts as a nonspecific suppressor of IL-8 secretion from CF cells. Consistently, pharmacogenomic analysis indicates that CF cells treated with CPX greatly resemble CF cells treated with CFTR by gene therapy. Additionally, the same result obtains in the presence of $P$. aeruginosa. Classical hierarchical cluster analysis, based on similarity of global gene expression, also supports this conclusion. The GENESAVER algorithm, using the IL-8 secretion level as a physiologic variable, identifies a subset of genes from the TNF- $\alpha \mathrm{R} / \mathrm{NF} \kappa \mathrm{B}$ pathway that is expressed in phase with IL-8 secretion from CF epithelial cells. Certain other genes, previously known to be positively associated with $\mathrm{CF}$, also fall into this category. Identified genes known to code for known inhibitors are expressed inversely, out of phase with IL-8 secretion.

Conclusions: Wild-type CFTR and CPX both suppress proinflammatory IL-8 secretion from CF epithelial cells. The mechanism, as defined by pharmacogenomic analysis, involves identified genes from the TNF- $\alpha \mathrm{R} / \mathrm{NF} \kappa \mathrm{B}$ pathway. The close relationship between IL- 8 secretion and genes from the TNF- $\alpha \mathrm{R} / \mathrm{NF} \kappa \mathrm{B}$ pathway suggests that molecular or pharmaceutical targeting of these novel genes may have strategic use in the development of new therapies for CF. From the perspective of global gene expression, both gene and drug therapy have similar genomic consequences. This is the first example showing equivalence of gene and drug therapy in $\mathrm{CF}$, and suggests that a gene therapy-defined endpoint may prove to be a powerful paradigm for $\mathrm{CF}$ drug discovery. Finally, because the GENESAVER algorithm is capable of isolating disease-relevant genes in a hypothesis-driven manner without recourse to any a priori knowledge about the system, this new algorithm may also prove useful in applications to other genetic diseases.
\end{abstract}

Address correspondence and reprint requests to: Harvey B.

Pollard, MD, PhD, Professor and Chair, Department of Anatomy, Physiology and Genetics, F. Edward Hebert School of Medicine School of Medicine, USUHS, 4301 Jones Bridge Road, Bethesda, MD 20814. Phone: (301) 295-3661; fax: (301) 295-2822; e-mail: hpollard@usuhs.mil

O. Eidelman and M. Srivastava contributed as co-equal first authors on this paper.

\section{Introduction}

Cystic fibrosis (CF) continues to be the most common fatal autosomal recessive disease in the United States, affecting 1 child per 2500 live births (1). Mutations in the chloride channel CFTR gene have been shown to be responsible for the disease, the most 
frequently occurring CF mutation being [ $\Delta \mathrm{F} 508$ ] CFTR (2-4). The consequences of this mutation are that the mutant protein fails to traffic efficiently through the endoplasmic reticulum to the plasma membrane where chloride conduction would otherwise occur (5-8). Recent attempts at pharmacologic treatment of CF have focused on using small molecules such as CPX (9), presently in phase 2 clinical trials, and phenylbutyrate (10) to promote proper trafficking of the mutant CFTR. This trafficking failure also has the functional consequence of establishing a pro-inflammatory environment by a mechanism that is poorly understood (11-14). Operationally, high levels of the pro-inflammatory cytokine interleukin-8 (IL-8) occur in the lung and are thought to be responsible for lung pathology in CF patients even prior to development of chronic bacterial infections $(15,16)$. The expectation is that IL-8 production in CF airway cells ought to be suppressed by wildtype CFTR or by CPX-enhanced trafficking of mutant CFTR. However, it is not known to what extent this conclusion is correct. Furthermore, the mechanisms by which CFTR might interact with the regulatory pathways controlling IL-8 synthesis and secretion are not known.

Using the CF airway epithelial cell line IB-3, we describe here mechanisms by which CFTR and CPX suppress IL-8 secretion. We further investigated the mechanism of this effect using cDNA microarray technology. We have previously used this approach to study the pharmacogenomics of CFTR and of the CF drug CPX in recombinant HEK293 fibroblasts expressing wild-type and $\Delta$ F508 CFTR (17), and this work extends these studies to natural CF lung epithelial cells. The reason for focusing on the gene microarray approach to the analysis of IL- 8 production by CF cells is that a large number of genes, including those from the NF $\kappa \mathrm{B}$ pathway, appear to be involved in the process (Fig. 1). For these reasons, we hypothesized that somewhere within the NF $\kappa \mathrm{B}$ pathway resides the mechanism responsible for the massive synthesis and secretion of pro-inflammatory IL-8 by CF lung epithelial cells.

Our present data indicate that both CFTR and CPX change the global gene expression of CF airway epithelial cells in similar ways, both in the presence

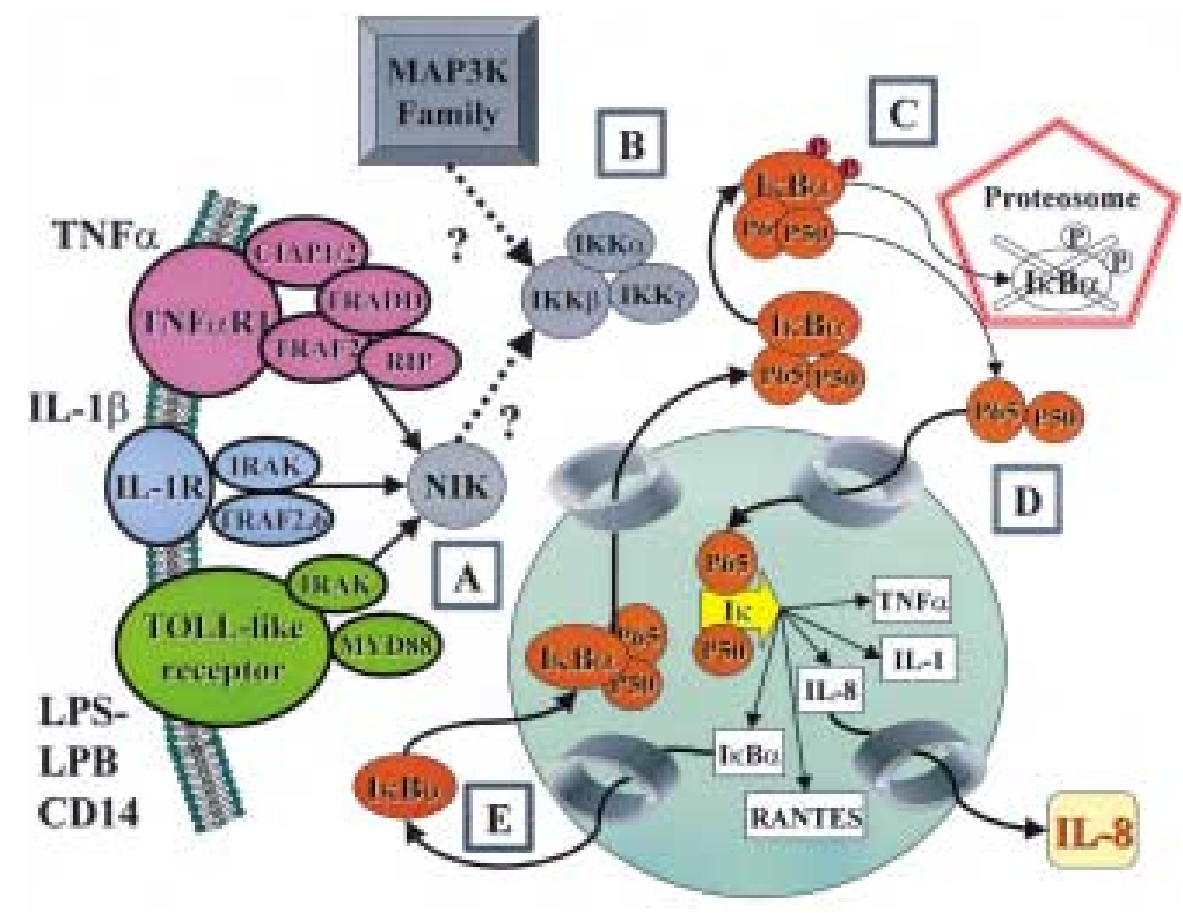

Fig. 1. The TNF- $\alpha$ R/NF $\kappa$ B signaling pathway. (A) The NF $\kappa$ B signaling cascade is hypothesized to be initiated by several independent pathways involving receptors for TNF- $\alpha$, IL-1, and bacterial lipopolysaccharides (LPS), which then converge on NIK, or on members of the MAP6K family. (B) Launching of this hypothetically common pathway activates a complex of I $\kappa \mathrm{B}-\mathrm{kinases}(\mathrm{IKK} \alpha$, $\beta$ and $\gamma$ ). The NF $\kappa$ B complex, composed of p65 and p50 components, is inactive when complexed with I $\kappa$ B. (C) When the activated IKKs phosphorylate $\mathrm{I} \kappa \mathrm{B}$, the proteosome attacks the phospho-I $\kappa \mathrm{B}$, releasing the residual p65/p50 complex. (D) This NF $\kappa \mathrm{B}$ heterodimer enters the nucleus and binds to $\kappa \mathrm{B}$ sites on promotors for IL-8, I $\kappa \mathrm{B}$, TNF- $\alpha$, IL-1, and other inflammatory signaling molecules. In this manner IL-8 gets transcribed and eventually secreted. It is worthy to note that this pathway induces both positive and negative feedback messages. TNF- $\alpha$ is an example of the former and $I \kappa B$ an example of the latter. (E) Once within the nucleus, the $\mathrm{NF} \kappa \mathrm{B}$ complex is removed from the $\kappa \mathrm{B}$ sites by binding to a fresh $\mathrm{I} \kappa \mathrm{B}$ molecule from the cytosol, which then leaves the nucleus as the inactive $\mathrm{p} 65 / \mathrm{p} 50 / \mathrm{I} \kappa \mathrm{B}$ complex. Under normal circumstances, the process then begins again upon physiologic activation. The $\mathrm{p} 50$ component is synthesized as a larger p105 precursor that is able to bind to p65. When bound to p65, p105 acts not only as a p50 ligand to p65, but also as an inhibitory I $\kappa \mathrm{B}-$ like ligand. In this latter role, the IKKs have no activating effect on the NF $\kappa \mathrm{B}$ complex [see Rothwarf and Karin (37)]. 
and absence of bacteria. Furthermore, using the novel GENESAVER algorithm, we find that genes involved in the TNF- $\alpha \mathrm{R} / \mathrm{NF} \kappa \mathrm{B}$ pathway are affected in similar ways by CFTR and CPX. The new algorithm also identifies genes known from the literature database to be associated with CF. We conclude that the tonic counter-inflammatory actions of CFTR, and of the CFTR surrogate CPX, in airway epithelial cells involve control of an ensemble of genes in the TNF- $\alpha \mathrm{R} / \mathrm{NF} \kappa \mathrm{B}$ pathway. We suggest that these new observations may be useful in developing novel approaches to drug discovery and therapy for CF.

\section{Materials and Methods}

\section{Culture of IB3 Cells and Assay of Secreted IL-8}

IB3 cells and repaired cell lines, IB3-S9 (repaired with AAV-wild-type CFTR) and IB3-C38 (repaired with AAV-[des 1-119]CFTR) were obtained from Dr. Pamela Zeitlin, Johns Hopkins University School of Medicine, and cultured in serum-free, gentimycinfree LHC-8 medium (Biofluids, Rockville, MD, USA). Unless otherwise specified, cells were grown to confluence in the presence or absence of CPX, washed with fresh medium, and then replaced in the incubator along with drugs or bacteria. Samples of supernatant medium were removed at the times shown and stored at $-80^{\circ} \mathrm{C}$ until assayed for IL-8. The IL-8 assay was performed using either a visible light enzyme-linked immunosorbent assay (ELISA) kit from Endogen (Woburn, MA, USA), or our ELISA system prepared from components supplied by Endogen. Results from both assays were equivalent.

\section{Culture of P. aeruginosa and Exposure to IB3 Cells}

A clinical isolate of $P$. aeruginos $a$ was obtained from the Food and Drug Administration (FDA). This isolate was determined to be a rough strain (personal communication, Dr. Joanna Goldberg, University of Virginia School of Medicine, Charlottesville, VA, USA), and, therefore, similar to strains isolated from CF patients. To analyze the infection of IB3 cells with $P$. aeruginosa, a modification of the cell association assay of Weinstein et al. (18) was used. Briefly, the bacteria were grown overnight in Luria Bertani (LB) medium at $37^{\circ} \mathrm{C}$ in a shaking water bath. One hundred microliters were then transferred to fresh LB and the bacteria grown to mid-late log. Subsequently, the bacteria were centrifuged at $10,000 \mathrm{rpm}$ for $10 \mathrm{~min}$, and then resuspended in an equivalent volume of LHC-8 medium. To determine the number of colony forming units in the culture, serial dilutions of the bacterial suspension were made in saline, and the appropriate dilutions were plated on LB agar plates. The IB3 monolayers were inoculated with P. aeruginosa at a multiplicity of infection of 15:1, and then spun onto the monolayer to promote maximal attachment. Incubation was allowed to proceed for $1.5 \mathrm{hr}$, after which the monolayers were washed extensively. Thereafter, LHC-8 with gentamicin $(100 \mu \mathrm{g} / \mathrm{ml})$ was added to remove extracellular bacteria. Supernatants were collected at specific times and cellular RNA was prepared from the IB 3 cells. The supernatants were stored at $-80^{\circ} \mathrm{C}$ until assay for IL-8.

Pharmacokinetics of CPX Action on IL-8 Secretion Duplicate samples of IB3 cells were plated with or without $10 \mu \mathrm{M}$ CPX in $0.1 \%$ DMSO and grown for $24 \mathrm{hr}$ in the LHC-8 medium. At the 24-hr time point, the medium was replaced with fresh medium with or without $10 \mu \mathrm{M}$ CPX, and the cells placed back in culture. As a control, a $0.1 \%$ DMSO vehicle replaced the CPX. At given times during the ensuing $24 \mathrm{hr}$, samples of supernatant from duplicate wells were taken for IL-8 ELISA assay. No significant differences in IL-8 secretion were noted between the $0.1 \%$ DMSO vehicle and medium without vehicle (see Fig. 1 inset). However, an inhibitory effect on IL-8 secretion was observed with $1 \%$ DMSO.

\section{Preparation of RNA for Genomic Analysis}

Total RNA was prepared from cells as described by Srivastava et al. (17). The total RNA preparation was then treated with DNAse I to eliminate genomic DNA contamination, and then treated with two rounds of purification of polyA + RNA using a mRNA isolation kit from Invitrogen (Carlsbad, CA, USA). The quality of the resultant mRNA was then be analyzed on a formaldehyde-denatured agarose gel, and by measurement of the OD260. To prepare ${ }^{32}[\mathrm{P}]-\mathrm{cDNA}$ probes corresponding to specifically known genes, $1 \mu \mathrm{g}$ of polyA + RNA was mixed with MMLV reverse transcriptase, dNTP mix, and cDNA synthesis primer mixes for the corresponding genes. The reaction was carried out on a thermocycler set at $50^{\circ} \mathrm{C}$ for $25 \mathrm{~min}$ and terminated by the addition of $0.1 \mathrm{M}$ of EDTA, $\mathrm{pH} 8.0$, and $1 \mathrm{mg} / \mathrm{ml}$ of glycogen. The ${ }^{32}[\mathrm{P}]$-labeled cDNA probes were then purified from unincorporated label on a CHROMASPIN-200 column (CLONTECH, Palo Alto, CA, USA).

\section{Labeling of cDNA Microarrays With Synthesized Probes}

The cDNA expression microarrays on nylon membranes were obtained from CLONTECH and were prehybridized using Express Hyb (CLONTECH) at $68^{\circ} \mathrm{C}$ for $\mathrm{l} \mathrm{hr}$, and then incubated overnight in Express Hyb with the denatured and neutralized labeled probes. Following this incubation, the membranes were washed three times in $2 \times \mathrm{SSC} / 1 \% \mathrm{SDS}$ at $68^{\circ} \mathrm{C}$ for $30 \mathrm{~min}$, and then twice in $0.1 \%$ SSC/0.5\% SDS at $68^{\circ} \mathrm{C}$ for $30 \mathrm{~min}$. The arrays were then exposed for $6 \mathrm{hr}$ on a Storm Phosphoimager plate (Molecular Dynamics, Sunnyvale, CA, USA).

\section{Imaging and Quantitation of the cDNA Microarray}

Imaging data from the Storm PhosphorImager was downloaded into a Microsoft Excel spreadsheet. Duplicate data were ratioed to the ubiquitin standard. Further validation was performed as described in Srivastava et al. (17). 


\section{Hierarchical Cluster Analysis of cDNA Array Data}

The clustering analysis was performed as described by Eisen et al. (19), and is accessible on the web at http://www.microarrays.org/software.

\section{GRASP Method for Data Mining From cDNA Arrays}

The strategy we employed is embodied in the GRASP methodology, in which variation in gene expression is quantitated in terms of the number of standard deviations (SDs) individual genes vary from the mean variation of the entire array [see Srivastava et al. (17)]. This technique vastly increases the statistical power of the analysis. Briefly, following ratioing to the ubiquitin standard, each gene on each blot is analyzed in duplicate, the error being about $1.3 \%$. Duplicate samples on duplicate blots were found to vary from each other by only about $12 \%$. Expression data for a given gene in a treated cell are then ratioed to expression in the parental cell line, and the log of this ratio is graphed against the $\log$ of an equivalent ratio of gene expressions for a different treatment to that of the parental cell line. The diagonal of this orthogonal plot is the distribution of equivalently expressing genes. The relationship of different genes to this diagonal is then expressed as being inside or outside the \pm 1 SD region around the diagonal. Relative positions on the orthogonal plot, outside the \pm 1 SD region then allow one to state that the expression of the given gene is or is not related to the mutation or experimental condition. The distance of each gene from the diagonal is then assessed as being a given multiple of SDs from the mean of the entire distribution.

\section{Genome Space Vector (GENESAVER) Method for Data Mining From cDNA Arrays}

For this study, we developed a novel data mining approach in which we calculate a seven-component vector based on the logarithms of the ratios of expression level of each gene in a certain condition relative to the expression level of that gene in a respective control condition. These seven conditions are (i) IB3 in the presence of CPX versus control IB3 cells; (ii) IB3 transfected with wt-CFTR (i.e., S9 cells) versus control IB3 cells; (iii) IB3 in the presence of $P$. aeruginosa versus control IB3 cells; (iv) IB3 in the presence of $P$. aeruginosa and CPX versus control IB3 cells; (v) S9 in the presence of CPX versus control S9 cells; (vi) S9 in the presence of $P$. aeruginosa versus control S9 cells; and (vii) S9 in the presence of P. aeruginosa and CPX versus control S9 cells. Similarly, we then calculate a vector describing the effects of these various experimental conditions on IL-8 secretion. We define each vector component as the log of the ratios of measured levels of secreted IL-8 in the experimental condition versus the respective control, as listed above. The next step is to calculate for each gene the angle (in seven-dimensional space) between its own vector and that of the IL-8 vector. We then sort the list of genes according to that calculated angle. This "genomic-space angle" reflects the correlation between the effects of these seven experimental conditions on IL-8 secretion and the expression levels of the given gene. As described, an angle close to $0^{\circ}$ defines genes whose expression closely parallels IL8 secretion in all conditions. An interesting additional feature of this approach is the ability to "mine out" anti-parallel (close to $180^{\circ}$ ) genes, whose pattern of expression is opposite to the pattern of IL-8 secretion. The latter genes are also of equal intrinsic interest because, a priori, we do not know whether a given gene has positive or negative actions on the process of IL-8 secretion. As a default correction for experimental and other errors, we used all genes within a $30^{\circ}$ solid angle of either $0^{\circ}$ or $180^{\circ}$. In addition, we only looked at genes that were significantly affected by the above conditions, by mining out those genes whose vectors were significantly greater than the average length (i.e., $\geq 1$ SD from the average vector length).

\section{Results}

\section{Influence of CFTR on Secretion of IL-8 From IB3 Cells}

IB3, IB3-S9, and IB-C38 cells were grown to confluence in 24 -well microtiter plates at $37^{\circ} \mathrm{C}$. At various times thereafter, the cell-free supernatants were assayed for the presence of IL-8 by ELISA. As shown in Figure 2, constitutive IL-8 secretion was very high in the CF mutant IB3 cells. In contrast, IL-8 constitutive secretion rates were substantially lower in IB3 cells repaired with wild-type CFTR, namely IB3-S9 cells. Over a 6-hr period, levels of IL-8 in the supernatants of IB3 cells were approximately $400 \mathrm{ng} /$ well compared to approximately $50 \mathrm{ng} /$ well for the IB3-S9 cells. Thus, the introduction of the wild-type CFTR gene into the mutant cells results in an 8-fold reduction in spontaneous IL-8 secretion. In addition we find that repair of IB 3 cells with a truncated form of CFTR, [des 1-119]-CFTR, also results in a partial suppression of IL-8 secretion. This truncated protein misses some binding sites for interaction with other proteins, but the chloride secretion function does not seem to be impaired (20). Furthermore, in IB3-C38 cells, NFkB-activation after treatment with TNF- $\alpha$ is distinct from that of IB3 cells, and seems to be commensurate with that of normal lung cells (21). These latter cells, IB3-C38, exhibit an approximately 5 -fold reduction in IL-8 released over the same 6-hr time period.

\section{Influence of the CFTR Activator CPX on IL-8 Secretion From IB3 Cells}

As shown in Figure 3, CPX reduces the rate of IL-8 secretion from IB 3 cells. After $24 \mathrm{hr}$ of treatment with $10 \mu \mathrm{M}$ of CPX, the rate of IL-8 secretion over the next $6 \mathrm{hr}$ was reduced by approximately 30\% 


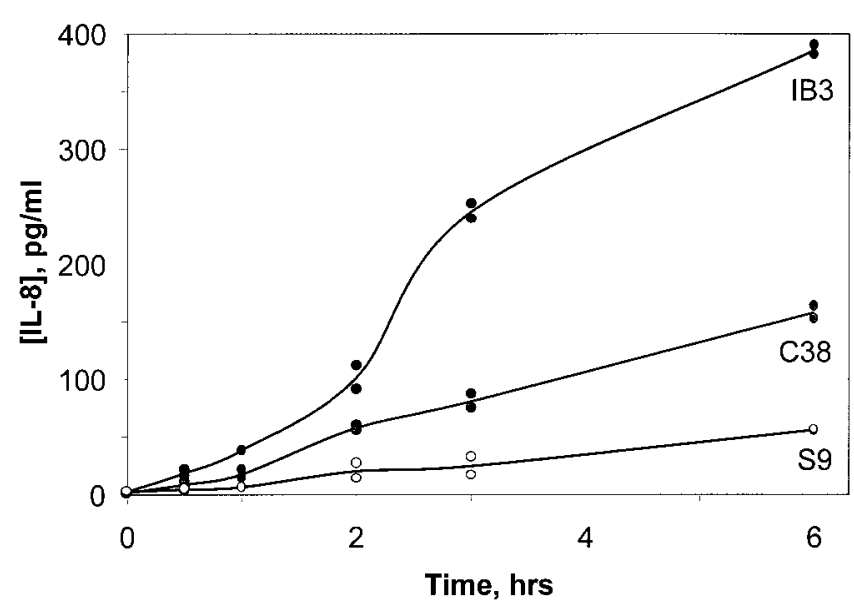

Fig. 2. Time course of IL-8 secretion from IB3 cells and CFTR transfected IB3 cells. IB3 cells and IB3 cells transfected either with wt-CFTR (S9) or with des[1-119]CFTR (C38) were grown to confluence in LHC-8 medium as described in Methods. At time 0, the medium was changed. Samples of the medium were taken at the indicated times and the IL-8 levels were determined by ELISA assay.

compared to the $0.1 \%$ DMSO control. This effect of CPX on IL-8 secretion is reversible, but lasted about $12 \mathrm{hr}$ after drug removal. The dashed gray line in Figure 3 shows that removal of CPX from the culture medium after the initial $24 \mathrm{hr}$ with $\mathrm{CPX}$ resulted in a sustained suppressive effect for up to $12 \mathrm{hr}$. However, the effect appeared to wash out eventually, resulting in control levels of IL-8 secretion by the $24-\mathrm{hr}$ time point. These rates are statistically different.

\section{Influence of P. aeruginosa and CPX on IL-8 Secretion From IB3 Cells}

In this experiment, cells were grown for $24 \mathrm{hr}$ in the presence or absence of $10 \mu \mathrm{M} \mathrm{CPX}$, washed, and then exposed to $P$. aeruginosa for an additional $1.5 \mathrm{hr}$ before cell-free supernatants were assayed for secreted IL-8. As shown in Figure 4, CPX alone reduces the level of constitutively secreted IL-8 in IB3 cells. This result is similar to that shown in Figure 3. In contrast, the effect of CPX on IL-8 secretion in the repaired IB3-S9 is less profound. When these cultures are subsequently incubated with $P$. aeruginosa several events occur. In the DSMO control cultures, $P$. aeruginosa induces IL-8 secretion in both the mutant CRTR and the repaired CFTR cell lines. Interestingly, in those cultures pretreated with CPX, P. aeruginosa induced more IL-8 secretion than in the DMSO control. Taken together, these data indicate that CPX suppresses constitutive levels of IL-8 secretion from the IB 3 cells, but does not interfere with the physiologic response of the IB 3 cells to the $P$. aeruginosa. In fact, the results suggest that CPX actually promotes the physiologic response of the CF epithelial cell to secrete more IL-8 in the presence of P. aeruginosa.

Gene Expression Analysis of CPX and CFTR Effects on IB3 Cells, in the Presence and Absence of P. aeruginosa To investigate the underlying genomic mechanisms of CPX and CFTR action on IB3 cells, we examined gene expression in these cells using cDNA microar-

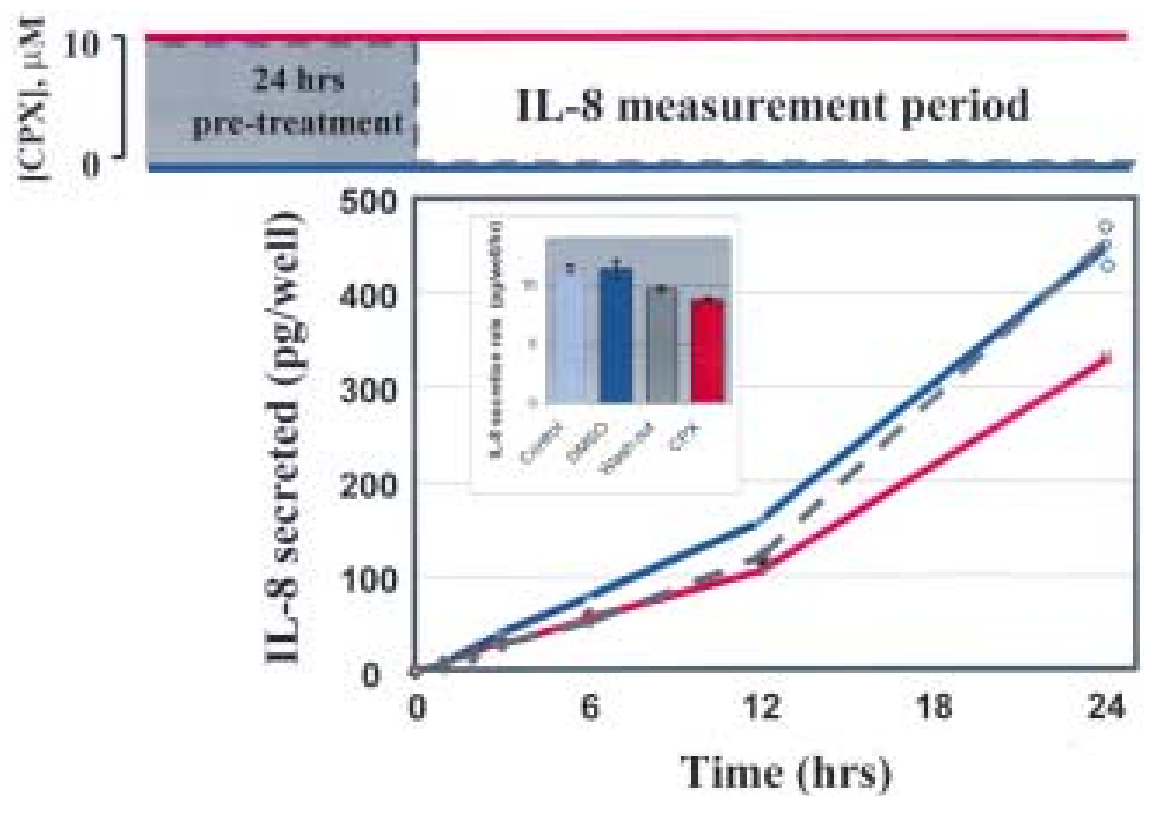

Fig. 3. The effect of CPX treatment on the time course of IL-8 secretion from IB3 cells. IB 3 cells were grown as described in Methods. Dishes labeled TREATMENT (red) or WASH-OUT (gray) were pretreated for 24 hr with $10 \mu \mathrm{M}$ CPX in LHC-8 and CONTROL (blue) dishes were pretreated with $0.1 \%$ DMSO. At time 0 , the media were changed to LHC-8 containing $10 \mu \mathrm{M}$ CPX (TREATMENT, red) or $0.1 \%$ DMSO (CONTROL, blue; WASH-OUT, gray). IL-8 levels were measured at the indicated time points. The top panel shows the levels of CPX for the respective samples during the pre-incubation and the measurement periods. The inset shows the rate of IL-8 secretion in the first $12 \mathrm{hr}$ calculated from the linear regression of the kinetic data. The bars show the rates for control without DMSO

(11.4 $\pm 0.4 \mathrm{pg} / \mathrm{well} / \mathrm{hr})$, control with DMSO (11.4 $\pm 0.8 \mathrm{pg} / \mathrm{well} / \mathrm{hr})$, CPX treatment $(8.8 \pm 0.2 \mathrm{pg} / \mathrm{well} / \mathrm{hr})$ and washout $(9.7 \pm 0.2 \mathrm{pg} / \mathrm{well} / \mathrm{hr})$. The IL-8-secretion rates of the Control and of the DMSOControl are virtually the same while the other rates are significantly different from each other at least at the $p<0.02$ level (CPX versus DMSO-Control: $p=0.0008$; Wash-Out versus DMSO-Control: $p=0.022$; Wash-Out versus CPX-treated: $p=0.0005)$. 


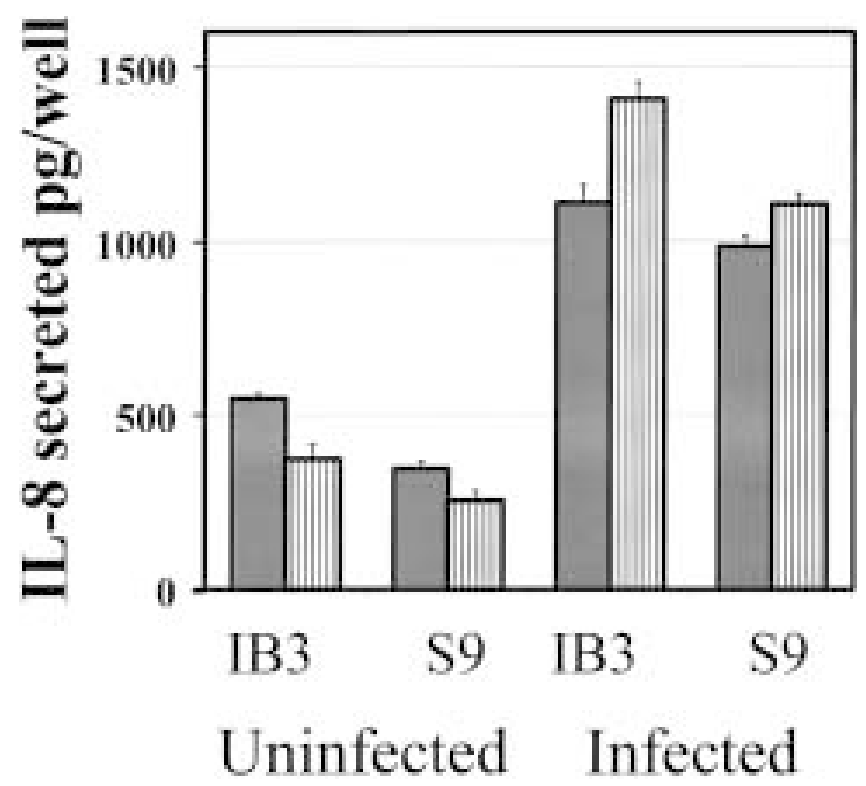

Fig. 4. The effect of CPX and of P. aeruginosa on IL-8 secretion from CFTR mutant IB3 cells and CFTR generepaired IB3-S9 cells. IB3 and IB3-S9 cells were grown as described in Methods in the presence of either $10 \mu \mathrm{M} \mathrm{CPX}$ (hatched bars) or $0.1 \%$ DMSO (solid bars), as indicated. The epithelial cells were then washed free of CPX or DMSO and incubated with P. Aeruginosa for $90 \mathrm{~min}$. The quantity of IL-8 secreted was then assessed by ELISA of the cell-free supernatant. Each CPX condition was found to be statistically different from its respective control $(p<0.05)$.

rays. For this purpose, we prepared RNA from the cells described in Figures 3 and 4 and used them for the gene expression analyses shown in Figures 5 and 6. These figures compare the expression level of each one of 588 genes in IB3 cells as a function of their expression level in the wild-type CFTRrepaired IB3-S9 cells. As shown in Figure 5, a substantial number of genes are expressed quite differently in IB3 and in IB3-S9 cells. This is evident by the points that are away from the main diagonal in each figure. The triangles in these graphs show the relation between gene expression in IB3 cells and in IB3-S9 cells. The circles depict the effects of culturing the IB3 cells in the presence of CPX for $48 \mathrm{hr}$ prior to RNA extraction. The effect of CPX on global gene expression in these cells can be assessed statistically by comparing the regression lines for all genes under the various conditions. By calculating the significance of the difference between the slopes of the regression lines we find that the CPX lines are significantly different than the no-CPX lines at the $p<0.001$ level, both in the presence (Fig. 6) and absence (Fig. 5) of $P$. aeruginosa. Because of our hypothesis regarding the possible importance of TNF- $\alpha \mathrm{R} / \mathrm{NF} \kappa \mathrm{B}-$ related genes, we have specifically marked the subset of TNF- $\alpha \mathrm{R} / \mathrm{NF} \kappa \mathrm{B}-$ related genes available in the array by large red triangles (without $\mathrm{CPX}$ ) or large blue circles (in the presence of CPX).

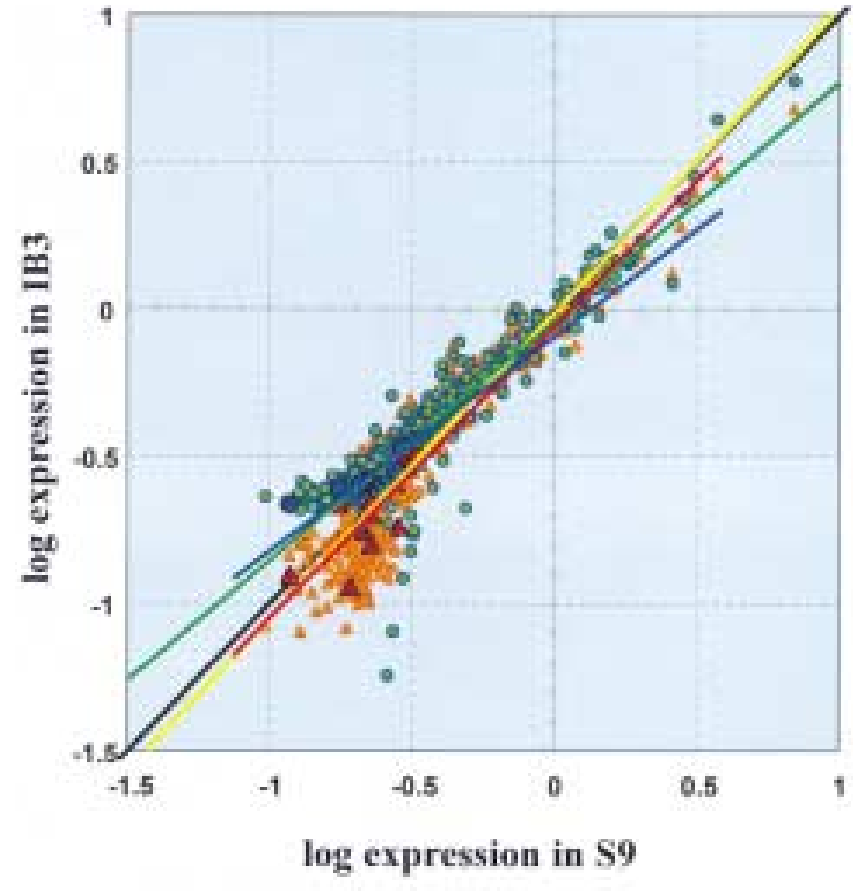

Fig. 5. Influence of CPX on global gene expression in CF IB3 and gene repaired IB3-S9 cells. Gene expression levels, plotted as a function of the expression levels of the respective genes in the wild-type CFTR transfected IB3-S9 cells, are compared between IB 3 cells (triangles) and CPX treated IB3 cells (circles). NF $\kappa \mathrm{B}$-related genes are denoted by larger symbols. The diagonal (black line) represents the location where expression level is equal to that in the IB3-S9 cells. The other lines are the linear regressions for the following groups: yellow, no CPX; red, NF $\kappa$ B-related genes with no CPX; green, with $10 \mu \mathrm{M}$ CPX; blue, NF $\kappa$ B-related genes with CPX. Note that when CPX is added to the cultures the expression of all genes in the array tends to move toward the diagonal.

Statistical analysis shows that the slopes of the regression line for the TNF- $\alpha \mathrm{R} / \mathrm{NF} \kappa \mathrm{B}$ subset is significantly different at the $p<0.05$ level from that of the TNF- $\alpha$ R/NF $\kappa$ B subset in cells cultured in the presence of CPX. However, in the presence of bacterial challenge from $P$. aeruginosa, the slopes of these two regression lines are not significantly different $(p=$ $0.5)$. These finding are in accord with the results shown in Figure 4, where CPX has an inhibitory effect on IL-8 secretion from IB3 cells when no bacteria are present, but little or even an increasing effect when challenged with $P$. aeruginosa.

Hierarchical Cluster Analysis of Gene Expression in IB3 Cells Following Exposure to CPX, CFTR, and P. aeruginosa As an alternative approach to understanding the genomic basis of common functional consequences for CPX and CFTR, we used hierarchical cluster analysis. This discovery-driven approach allows us to ask which genes and which cell conditions are most correlated without specific reference to our own a priori assumptions. Our approach to this analysis has been to use gene expression ratios for 


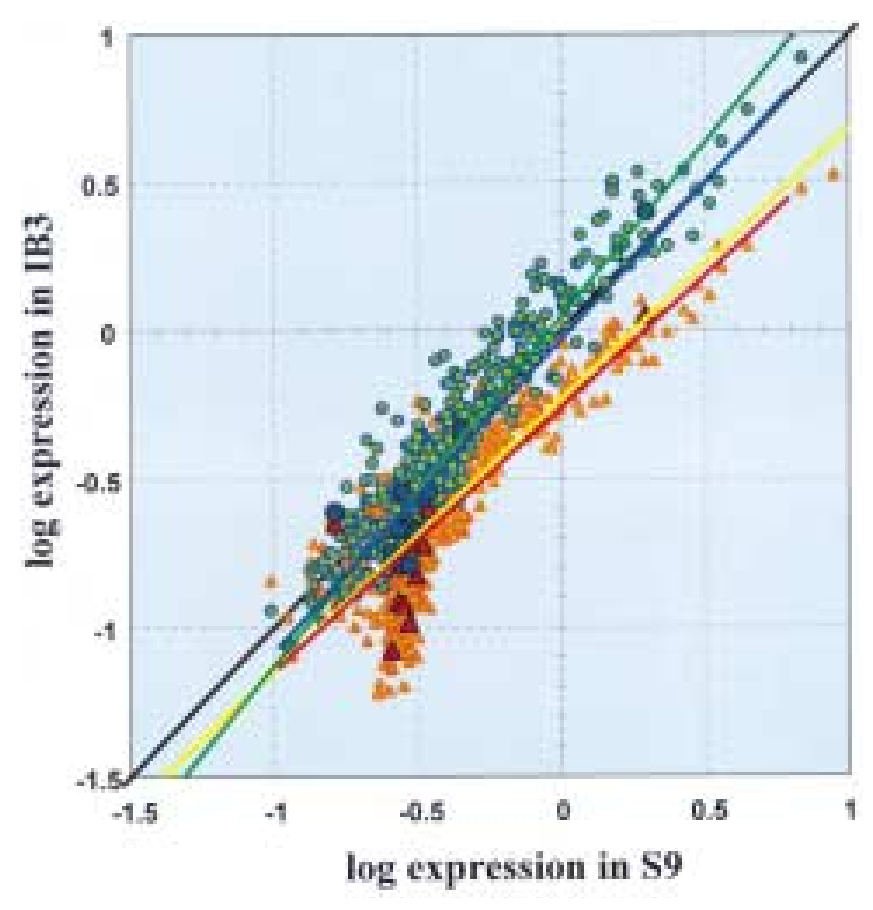

Fig. 6. Influence of CPX on global gene expression of CF IB3 and gene repaired IB3-S9 cells exposed to $P$. aeruginosa. Gene expression levels in IB3 cells exposed to P. aeruginosa for $1.5 \mathrm{hr}$ are plotted as a function of the expression levels of the respective genes in the wild-type CFTR transfected IB3-S9 cells. The symbols and the colors are as described in the legend to Figure 5.

IB3 cells under conditions of with or without CPX, CFTR, and P. aeruginosa, and to allow the clustering algorithm to dyadically group cells and genes.

As shown in Figure 7, the clustering of the experimental conditions (top dendritic tree) indicates several discrete dyadic clusters. The first such dyadic cluster includes repaired IB3-S9 cells and IB3 cells treated with CPX. This result indicates that the clustering algorithm finds that CPX treatment makes the mutant cells genomically more similar to the CFTRrepaired IB3-S9 cells than to the untreated parental IB3 cells themselves. The dyadic cluster on the extreme left indicates that the same is true in the presence of $P$. aeruginosa. The third dyadic cluster is IB3 cells in the presence or absence of $P$. aeruginosa. This result indicates that the clustering algorithm finds that IB3 cells respond to the bacteria so modestly that their patterns of gene expression are virtually identical. This might be indicative of the fact that the IB3 cells are already in a "stimulated" state, even without bacteria present. A fourth dyadic cluster consists of IB3-S9 cells with added bacteria, in the presence or absence of CPX. This result indicates that CPX confers little additional difference to the genomic response of CFTR-repaired IB3 cells when challenged by exposure to P. aeruginosa.

The genes themselves (the vertical left hand dendritic tree) cluster into at least 13 major groups.
These clusters can be studied to determine whether $\mathrm{NF} \kappa \mathrm{B}$ pathway genes are multiply associated with any of these clusters. One specific question we asked was whether the activating $\mathrm{p} 65 \mathrm{NF} \kappa \mathrm{B}$ subunit or the inhibitory p $105 \mathrm{NF} \kappa \mathrm{B}$ subunit clustered together. As shown in Figure 7, we found that p65 and p105 occurred in separate clusters, and that both clustered separately from the presumably unrelated $\mathrm{R} \kappa \mathrm{B}$ gene. Other genes, not clearly related to either p65 or p105, also clustered with each of these species. Thus it became clear that although we could functionally distinguish identified genes from one another by this approach, it was less feasible to use this method to make a compelling positive identification of functionally related genes. In addition, because there are approximately as many $\mathrm{NF} \kappa \mathrm{B}$-related genes as primary clusters, it is clear that several of these NF $\kappa$ B genes might, by sheer coincidence, group into one of the clusters. Thus, a more physiologically relevant method was needed for analyzing the clustering patterns of these large ensembles of genes.

Development of a Physiology-Based Vectorial Cluster Analysis of Gene Expression in IB3 Cells

Gene expression by cells under a variety of conditions can also be analyzed by clustering according to a physiologic pattern. In the present case, we asked which genes in IB3 cells paralleled the pattern of expression and secretion of IL-8. Although this concept is simple, to achieve this aim we had to develop a novel bioinformatics paradigm that would allow us to assess the correlation between expression patterns and experimental results. Our approach was to assign for each gene a "genomic space vector" based on the gene's response to various experimental conditions. Using these vectors we then calculated for each gene a "genomic space angle" between the gene's vector and a similar vector based on the physiologically relevant parameter of IL-8 secretion.

The rationale behind this approach is that the smaller the angle between the two vectors, the more closely the expression pattern of the gene of interest correlated with the pattern of expression and secretion of IL-8. Thus, an angle of $0^{\circ}$ would mean exact convergence. Exact convergence means that when IL-8 secretion went up, the expression level of the gene of interest would also go up and vice versa, in phase with the IL-8 activity. By contrast, an angle of $180^{\circ}$ would mean an exactly inverse behavior, so that when IL-8 secretion went up, the expression level of the gene of interest would go down, and vice versa. We also used the GRASP technique (17) to mine out those genes that both satisfied the angle criteria and were more than I SD greater than the logarithmic average length of all the vectors. We have called this new datamining algorithm GENESAVER (Genomic Space Vector). The details of this procedure are given in the Methods section. 


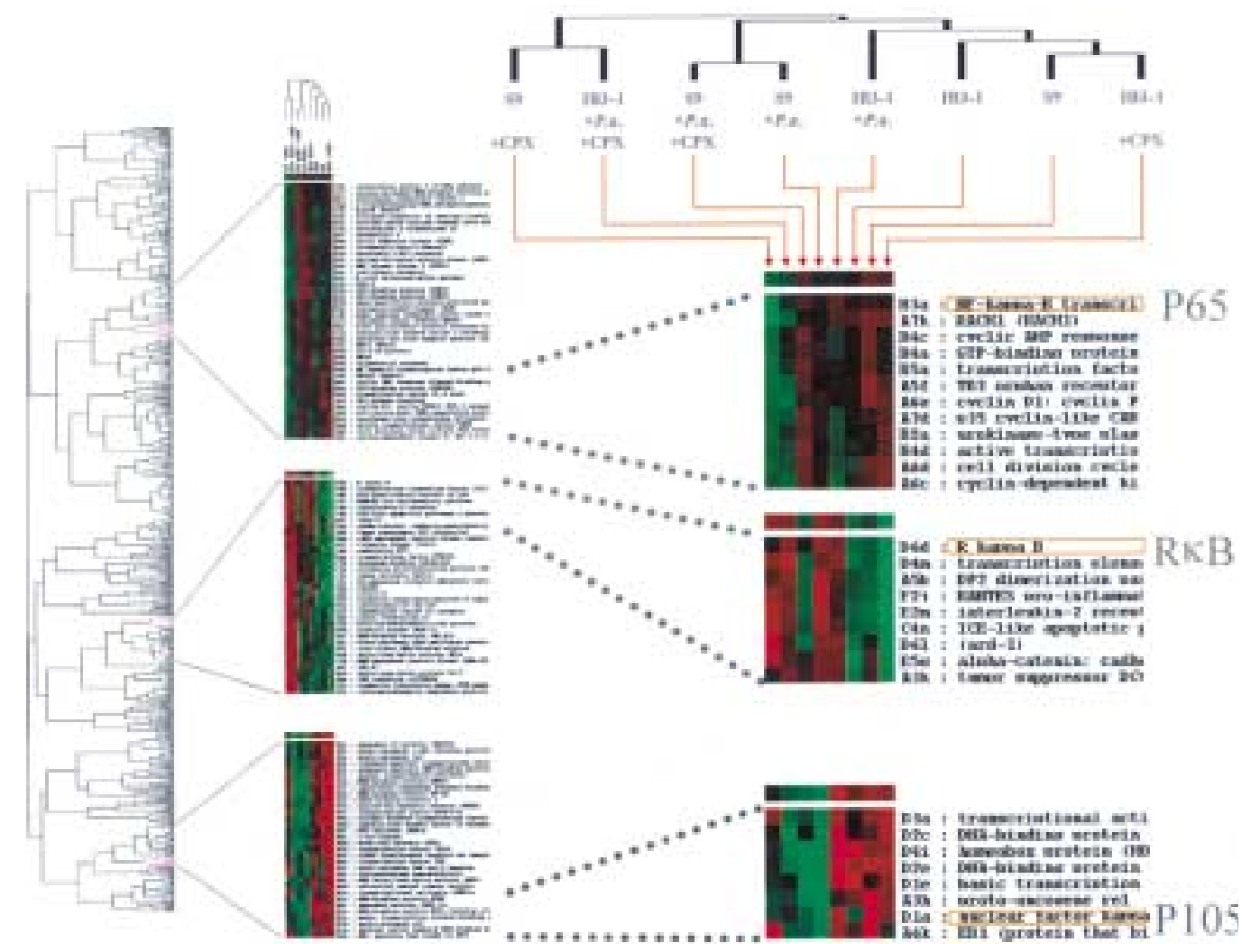

Fig. 7. Hierarchical Cluster Analysis of genes and of experimental conditions for IB3 cells. Gene expression levels for all 588 genes (shown on the left) in the eight experimental conditions shown on the top dendritic tree were analyzed using the cluster and the tree view applications from Stanford (www.microarrays.com). On the horizontal axis, the far-right dyad shows that treatment of the CF IB3 cells with CPX causes it to most closely resemble the gene repaired IB3-S9 cell. Other relationships are discussed in the text. On the vertical axis, relative expression of genes are color-coded in graded red (high expression) and graded green (low expression) The indicated parts of the gene expression tree are magnified in the middle panel and further enlarged in the right panel. Note that NF $\kappa \mathrm{B} / \mathrm{p} 65$, which activates IL-8 production, and NF $\kappa \mathrm{B} / \mathrm{p} 105$, which suppresses IL-8 production, cluster separately from each other, and from R $\kappa$ B and RANTES.

The significant data from this bioinformatics experiment are abstracted in Table 1. For each gene, we give the array location, the significance of the change (in standard deviations from the average change), and the angle in seven-dimensional space. These data show that certain pro-inflammatory or pro-apoptotic genes are positively and significantly correlated with IL-8 secretion by IB3 cells under the eight experimental conditions (see Fig. 1 for relationships). These include the TNF- $\alpha$ receptor itself, and the TNF- $\alpha$ receptor adapter proteins TRADD, CIAPI, RIP, TRAF (CRAFl), and TRAMP. The remainder includes five TNF- $\alpha$ receptor-associated pro-apoptotic genes, as well as RANTES and TGF $\beta$. Six other positively correlated genes are listed separately, which as a group are associated with either cell division or cellular proliferation.

of the genes whose expression is negatively and significantly correlated with IL-8 secretion, we found only one NF $\kappa \mathrm{B}$-related gene: the inhibitory $\mathrm{NF} \kappa \mathrm{B}-$ p105 subunit. However, in addition to NFkB-p105, there was also a surprisingly familiar face: the gene for ezrin. This gene is an adapter protein that binds to CFTR through PDZ domains linkers, and is known to be important for processing and apical targeting of CFTR. Finally, within this negatively correlated group is a set of 13 genes associated with either cell division or transcription. We have calculated that the probability of this number of identified genes occurring purely by chance within a $\pm 60^{\circ}$ solid angle in seven-dimensional space can be as low as $1.3 \times 10^{-10}$ (i.e., $\sim 1$ chance in 10 billion). In an effort to be complete, we also list in our analysis those genes in the array that, although not correlating well with IL-8 secretion, do fall into the top $5 \%$ of the distribution of gene vector lengths. The complete data for all genes available to us in our arrays can be accessed on our web site (http://www.usuhs.mil).

\section{Discussion}

Using pharmacogenomic and biochemical techniques to analyze IB3 CF airway epithelial cells, we have determined that the typically high levels of secretion of the pro-inflammatory cytokine IL-8 can be suppressed by repair with either wild-type CFTR, or by treatment with the xanthine drug CPX. Our data indicate that the mechanism of IL-8 control in CF cells 
Table 1. Correlation between gene expression and IL8 secretion using the genomic space vectors (GENESAVER) algorithm

\begin{tabular}{|c|c|c|c|}
\hline $\begin{array}{l}\text { Gene } \\
\text { Description }\end{array}$ & $\begin{array}{c}\text { Positive Correlation } \\
\left(\mathbf{( 0 - 6 0 ^ { \circ } )}\right. \\
\text { [Location, S.D., Angle] }\end{array}$ & $\begin{array}{c}\text { Inverse Correlation } \\
\left(120-180^{\circ}\right) \\
\text { [Location, S.D., Angle] }\end{array}$ & $\begin{array}{c}\text { Not Correlated } \\
\left(60^{\circ}-120^{\circ}\right) \\
\text { [Location, S.D., Angle] }\end{array}$ \\
\hline $\begin{array}{l}\text { Associated with TNF } \\
\text { Receptors and } \\
\mathrm{NF} \kappa \mathrm{B} \text { pathway }\end{array}$ & 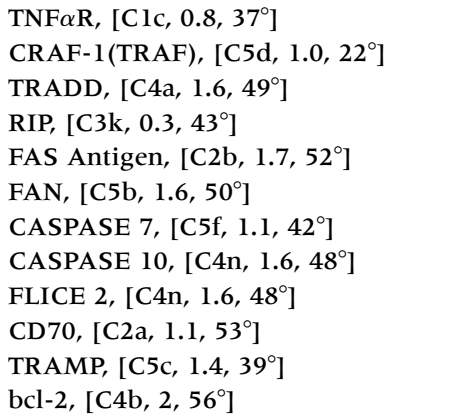 & $\mathrm{NF} \kappa \mathrm{B}-\mathrm{p} 105,\left[\mathrm{Dla}, 1.3,128^{\circ}\right]$ & \\
\hline Other Inflammatory & $\begin{array}{l}\text { RANTES, }\left[F 2 \mathrm{j}, 1.4,52^{\circ}\right] \\
\text { TGF } \beta,\left[C 1 f, 1.0,23^{\circ}\right]\end{array}$ & Interferon consensus $\mathrm{BP},\left[\mathrm{D} 1 \mathrm{j}, 2.3,147^{\circ}\right]$ & \\
\hline Known to bind CFTR & & EZRIN, [A2f, 1.2, $\left.143^{\circ}\right]$ & \\
\hline Other (Top $5 \%$ by length) & 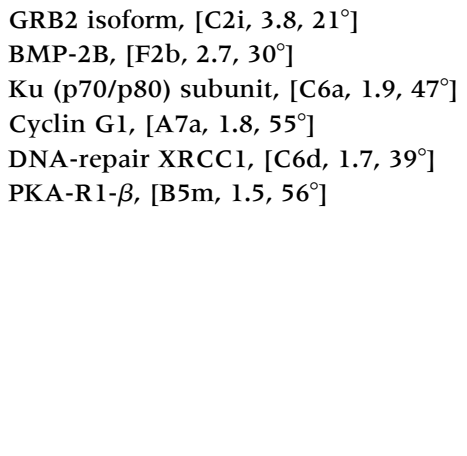 & 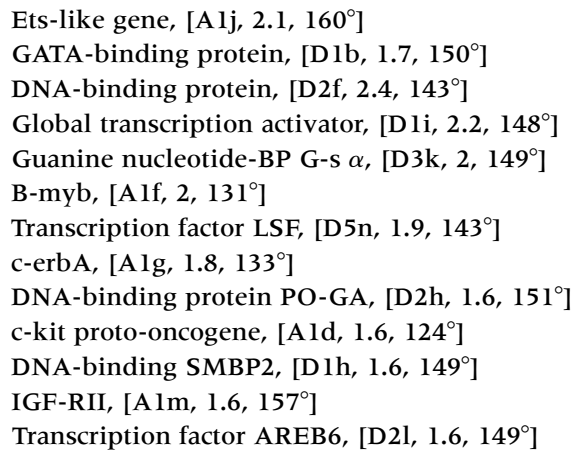 & $\begin{array}{l}\text { IGF-IR, }\left[\mathrm{Cla}, 2.3,67^{\circ}\right] \\
\text { c-myb } \lambda,\left[\text { Alc, } 2.2,116^{\circ}\right] \\
\text { SLAP, }\left[\mathrm{B} 6 \mathrm{a}, 2,70^{\circ}\right] \\
\text { Ca-ATPase } 2,\left[\mathrm{~B} 1 \mathrm{a}, 1.9,89^{\circ}\right] \\
\text { i NOS, [Clb, 1.9,63 }] \\
\text { NIP1 (NIP1), }\left[\mathrm{C} 4 \mathrm{c}, 1.8,63^{\circ}\right]\end{array}$ \\
\hline
\end{tabular}

involves a direct relationship between wild-type CFTR protein expression, regulated IL-8 expression, and mRNA expression of an ensemble of genes from the TNF- $\alpha$ R/NF $\kappa$ B pathway. Briefly, the presence of mutant CFTR results in elevation of IL-8 expression, which is paralleled genomically by an elevation in a subset of activating genes in the TNF- $\alpha \mathrm{R} / \mathrm{NF} \kappa \mathrm{B}$ pathway, or by reduction in certain inhibitory genes from the same pathway. Therefore, we interpret these data to suggest that molecular or pharmaceutical targeting of these ensembles of identified genes may have strategic use in developing new therapies for CF.

A New Pharmacogenomic Paradigm for Drug Discovery in $C F$

The rationale behind a pharmacogenomic approach to CF drug discovery has been the expectation that an effective drug could induce a CF cell to behave genomically similar to one treated with wild-type CFTR. In the case of the drug CPX, this expectation has now been realized. To achieve this end, a classical hierarchical clustering algorithm was utilized to test this hy- pothesis. This algorithm (see Fig. 7) allowed us to scrutinize binary comparisons of all the conditions in terms of similarity of gene expression for all genes in the array. Without knowing the identity of specific genes, the algorithm sorted through all possible arrangements of gene expression and determined that cells treated with wild-type CFTR (i.e., IB3-S9) or with CPX more closely resembled each other than they resembled the parental IB3 cells. This observation is the first example of which we are aware where one can actually demonstrate the premise of the pharmacogenomics paradigm for drug discovery.

We also used this approach to model drug and gene actions on the response of CF cells to exposure to $P$. aeruginosa. These experiments are important because many CF patients are chronically infected with this gram-negative bacterium. In genomic experiments using the GRASP algorithm, we found that IB3 cells responded quite modestly to exposure to a clinical isolate of this organism. However, IB3 cells treated with either wild-type CFTR or CPX responded robustly and similarly, with significant movement noted for 
$\mathrm{NF} \kappa \mathrm{B}-$ related genes. This result was borne out independently when the hierarchical clustering algorithm was used to analyze the entire gene array. Thus, we conclude that CPX and CFTR induce similar changes in gene expression, and that similar, but additional, changes occur in the presence of bacteria. We also conclude from this set of results that the CF cell appears to have a fundamental problem in responding to the presence of $P$. aeruginosa. One possible basis for this phenomenon is that the simple presence of the CFTR mutation induces the cell's control systems to act as though bacteria have already arrived. If our idea is correct, then such a result would mean that the CF cell has already launched a massive baseline pro-inflammatory response and that further relevant responses at the genomic level are not possible. The genomic basis of this pathophysiologic process seems to be tonic activation of the TNF- $\alpha \mathrm{R} / \mathrm{NF} \kappa \mathrm{B}$ pathway.

\section{Development of the GENESAVER Algorithm to Identify Genes on the Basis of a Physiologic Variable}

To be able to draw conclusions about the identity of genes involved in the enhanced constitutive secretion of IL-8 by CF lung cells, we needed to develop the GENESAVER algorithm. GENESAVER uses a multidimensional analysis paradigm to determine exactly which genes in the entire array change in parallel with the physiologic variable of IL-8 activity. In fact, we had originally hypothesized that some of the genes from the $\mathrm{NF} \kappa \mathrm{B}$ pathway might be important. After all, there has been a well-documented relationship between IL-8 expression and control by the NF $\kappa$ B system in epithelial cells. However, this pathway is so complex, and the cross-talk with other pathways so extensive (see Fig. 1) that we needed to develop an analytic approach that did not take this prior knowledge into account. The development of the GENESAVER algorithm allowed us to use the physiologic variable IL-8 as a compass to tease out only those genes that precisely followed IL-8 secretion, either positively or negatively. The validation of this approach rests in the fact that the algorithm identifies genes from the TNF- $\alpha \mathrm{R}$ and $\mathrm{NF} \kappa \mathrm{B}$ systems, which have historically been intimately linked in the literature database with the CF phenotype. Importantly, however, as shown below, this algorithm also identifies other genes with a historical association with CF.

Validation of GENESAVER-Identified Genes by Recourse to the Literature Database

This database linkage includes the relationship between CF and apoptotic signaling. For example, a 10-fold elevation in TUNEL labeling for fragmented DNA has been reported in crypt enterocytes observed in duodenal biopsies of CF patients (24). In addition, expression of FAS and FAS ligand proteins, both markers of apoptosis, are markedly increased in biopsies of CF bronchial epithelium and cultured CF tracheal cell lines (25). Finally, disordered regulation of apoptosis has also been reported in heterologous C127 cells expressing mutant [DF508]CFTR (26). However, the other apoptotic genes identified by the GENESAVER algorithm have not previously been associated specifically with CF. These include TNF- $\alpha$ receptor- 1 associated death domain protein (TRADD), TNF- $\alpha$ receptor associated factor (TRAF2), TNF- $\alpha$ receptor interacting protein (RIP), and inhibitor of apotosis protein 1 (CIAP1/2). These genes are involved in transducing interactions between TNF- $\alpha \mathrm{R}$ and downstream apoptotic components such as caspase 7, FLICE, FAS antigen, FAN, and TRAMP. Therefore, it is a reasonable expectation that if the downstream factors are important, then upstream adapters will probably be important as well. Nonetheless, the significance of any of these identified genes for CF remains a subject for further investigation.

The power of the GENESAVER algorithm is further manifest by its ability to identify additional genes with substantial CF pedigrees (see Table 1). For example, RANTES and TGF- $\beta$ are identified as positively correlated with IL-8 secretion, whereas ezrin is inversely correlated. In the case of RANTES, it is known that production of this cytokine by CF epithelial cells is affected by $P$. aeruginosa exotoxins such as pyocyanin (27) and exoenzyme $S$ (28), as well as the presence of wild-type CFTR in cultured epithelial cells (29). High levels of TGF- $\beta 1$ are expressed in the hyperplastic epithelium and elsewhere in CF patients (30). In addition, a high TGF- $\beta$ producer genotype is positively correlated in CF patients with increased risk of accelerated decline in pulmonary function (31). Finally, very high expression levels of TGF- $\beta$, and incidentally, also of interferon- $\gamma$, have been reported in CF patients (32). Ezrin has been implicated with not only targeting of CFTR to the apical plasma membrane of lung epithelial cells $(33,34)$, but also with interaction between CFTR and protein kinase A $(35,36)$. It is possible that as IL-8 secretion is suppressed by elevated CFTR expression, ezrin may be induced in parallel to provide the escort service for CFTR to the plasma membrane. Finally, we are unaware of any specific relationship to CF of those genes identified in the category of cell division and transcription. However, as further study and evaluation of these pharmacogenomic results progress, the importance of some of these genes could become apparent.

\section{Conclusions}

Wild-type CFTR and CPX both suppress proinflammatory IL-8 secretion from CF epithelial cells. The mechanism, as defined by pharmacogenomic analysis, involves identified genes from the TNF- $\alpha$ R/NF $\kappa$ B pathway. The close relationship between IL-8 secretion and genes in the TNF- $\alpha \mathrm{R} / \mathrm{NF} \kappa \mathrm{B}$ pathway suggests that molecular or pharmaceutical targeting of these novel genes may have strategic use for the development of new therapies for CF. From the perspec- 
tive of global gene expression, both gene and drug therapy have similar genomic consequences. Our results are the first example showing equivalence of gene and drug therapy in CF. Our findings suggest that a gene therapy-defined endpoint may prove to be a powerful paradigm for CF drug discovery. Finally, because the GENESAVER algorithm is capable of isolating disease-relevant genes in a hypothesis-driven manner, without recourse to any a priori knowledge about the system, this new algorithm may also prove useful in applications to other genetic diseases.

\section{Acknowledgments}

The authors gratefully acknowledge Ms. Bette Pollard for reading and editorial comments on the manuscript. This work was supported in part by grants from the NIH (RO1-DK53051 to H.B.P.), (R01-AI48151 to E.S.M), and the Cystic Fibrosis Foundation (H.B.P.).

\section{References}

1. Welsh MJ, Ramsey BW, Accurso F and Cutting G. (2001) Cystic Fibrosis. In Scriver CL, Beaudet AL, Valle D, Sly WS, eds. The Metabolic and Molecular Bases of Inherited Disease, 8th ed. New York: McGraw-Hill pp. 5121-5188.

2. Rommens JM, Lannuzzi MC, Karem B-S, et al. (1989) Identification of the cystic fibrosis gene: chromosome walking and jumping. Science 245: 1059-1065.

3. Riordan JR, Rommens JM, Karem B-S, et al. (1989) Identification of the cystic fibrosis gene: Cloning and characterization of complementary DNA. Science 245: 1066-1073.

4. Kerem B-S Rommens JM, Buchanan JA, et al. (1989) Identification of the cystic fibrosis gene: genetic analysis. Science 245: 1073-1080.

5. Cheng SH, Gregory RJ, Marshall J, et al. (1990) Defective intracellular transport and processing of CFTR is the molecular basis of most cystic fibrosis. Cell 63: 827-834.

6. Lukacs GL, Mohamed A, Kartner N, Chang X-B, Riordan JR, Grinstein S. (1994) Conformational maturation of CFTR but not its mutant counterpart $(\triangle \mathrm{F} 508)$ occurs in the endoplasmic reticulum and requires ATP. EMBO J. 13: 6076-6086.

7. Ward CL, Kopito RR. (1994) Intracellular turnover of Cystic Fibrosis Transmembrane Conductance Regulator. Inefficient processing and rapid degradation of wild type and mutant protein. J. Biol. Chem. 269: 25710-25718.

8. Yang Y, Janich S, Cohn JA, Wilson JM. (1993) The common variant of cystic fibrosis transmembrane conductance regulator is recognized by hsp70 and degraded in a pre-Golgi nonlysosomal compartment. Proc. Natl. Acad. Sci. U.S.A. 90: 94809484.

9. Eidelman O, Guay-Broder C, van Galen PJM, et al. (1992) Al adenosine-receptor antagonists activate chloride efflux from cystic fibrosis cells. Proc. Natl. Acad. Sci. U.S.A. 89: 5562-5566.

10. Rubenstein RC, Zeitlin PL. (2000) Sodium 4-phenylbutyrate downregulates Hsc70: implications for intracellular trafficking of SF508-CFTR. Am. J. Physiol. Cell Physiol. 278: C259C267.

11. Dean TP, Dai Y, Shute JK, Church MK, Warner JO. (1993) Interleukin-8 concentrations are elevated in bronchoalveolar lavage, sputum and sera of children with cystic fibrosis. Pediatr. Res. 34: 159-161.

12. DiMango E, Ratner AJ, Bryan R, Tabibi S, Prince A. (1998) Activation of $\mathrm{NF} \kappa \mathrm{B}$ by adherent Pseudomonas aeruginosa in normal and cystic fibrosis respiratory epithelial cells. J. Clin. Invest. 101: 2598-2605.

13. Francoeur C, Denis M. (1995) Nitric oxide and interleukin-8 as inflammatory components of cystic fibrosis. Inflammation 19: 587-598.
14. Richman-Eisenstat JB, Jorens PG, Hebert CA, Ueki I, Nadel JA. (1993) Interleukin-8: an important chemoattractant in sputum of patients with chronic inflammatory airway diseases. Am. J. Physiol. 264: L413-L418.

15. Briars GL, Dean TP, Murphy JL, Rolles CJ, Warner JO. (1995) Faecal interleukin-8 and tumour necrosis factoralpha concentrations in cystic fibrosis. Arch. Dis. Child. 73: 74-76.

16. Khan TZ, Wagener JS, Bost T, Martinez J, Accurso FJ, Riches DW. (1995) Early pulmonary inflammation in infants with cystic fibrosis. Am. J. Respir. Crit. Care Med. 151: 1075-1082.

17. Srivastava M, Eidelman O, Pollard HB. (1999) Pharmacogenomics of the Cystic Fibrosis Transmembrane Conductance Regulator (CFTR) and the Cystic Fibrosis Drug CPX using genome microarray analysis. Mol. Med. 5: 753-767.

18. Weinstein DL, O'Neill B, Metcalf ES. (1997) Salmonella typhi stimulation of human intestinal epithelial cells induces secretion of epithelial cell-derived interleukin-6. Infect. Immun. 65: 395-404.

19. Eisen MB, Spellman PT, Brown PO, Botstein D. (1998) Cluster analysis and display of genome-wide expression patterns. Proc. Natl. Acad. Sci. U.S.A. 95: 14863-14868.

20. Egan M, Flotte T, Afione S, et al. (1992) Defective regulation of outwardly rectifying $\mathrm{Cl}$-channels by protein kinase A corrected by insertion of CFTR. Nature 358: 581-584.

21. Venkatakrishnan A, Stecenko AA, King G, et al. (2000) Exaggerated activation of nuclear factor- $\kappa \mathrm{B}$ and altered $\mathrm{I} \kappa \mathrm{B}-\beta$ processing in cystic fibrosis bronchial epithelial cells. Am. J. Respir. Cell. Mol. Biol. 23: 396-403.

22. Casavola V, Turner RJ, Guay-Boder C, Jacobson KA, Eidelman O, Pollard HB. (1995) CPX, a selective Al-adenosine-receptor antagonist, regulates intracellular $\mathrm{pH}$ in cystic fibrosis cells. Am. J. Physiol. 269: C226-C233.

23. Arispe N, Ma J, Jacobson KA, Pollard HB. (1998) Direct activation of Cystic Fibrosis Transmembrane Conductance Regulator by 8-cyclopentyl-1,3-dipropylxanthine and 1,3Diallyl-8-cyclohexylxanthine. J. Biol. Chem. 273: 5724-5734.

24. Maiuri L, Raia V, De Marco G, et al. (1997) DNA fragmentation is a feature of cystic fibrosis epithelial cells: a disease with inappropriate apoptosis? FEBS Lett. 408: 225231.

25. Durieu I, Amsellem C, Paulin C, et al. (1999) Fas and Fas ligand expression in cystic fibrosis airway epithelium. Thorax 54: 1093-1098.

26. Gottlieb RA, Dasanjh A. (1996) Mutant cystic fibrosis transmembrane conductance regulator inhibits acidification and apoptosis in C127 cells possible relevance to cystic fibrosis. Proc. Natl. Acad. Sci. U.S.A. 93: 3587-3591.

27. Denning GM, Wollenweber LA, Railsback MA, Cox CD, Stoll LL, Britigan BE. (1998) Pseudomonas pyocyanin increases interleukin-8 expression by human airway epithelial cells. Infect. Immun. 66: 5777-5784.

28. Epelman S, Bruno TF, Neely GG, Woods DE, Mody CH. (2000) Pseudomonas aeruginosa exoenzyme $S$ induces transcriptional expression of pro-inflammatory cytokines and chemokines. Infect. Immun. 68: 481 1-4814.

29. Schweibert LM, Estell K, Propst SM. (1999) Chemokine expression in CF epithelia: implications for the role of CFTR in RANTES expression. Am. J. Physiol. 276: C700-C710.

30. Corrin B, Butcher D, McAnulty BJ, et al. (1994) Immunohistochemical localization of transforming growth factor-beta 1 in the lungs of patients with systemic sclerosis, cryptogenic fibrosing alveolitis and other lung disorders. Histopathology 24: 145-150.

31. Arkwright PD, Laurie S, Super M, et al. (2000) TGF-beta(1) genotype and accelerated decline in lung function in patients with cystic fibrosis. Thorax 55: 459-462.

32. Wojnarowski C, Frischer T, Hofbauer E, et al. (1999) Cytokine expression in bronchial biopsies of cystic fibrosis patients with and without acute exacerbation. Eur. Respir. J. 14: 1136-1144.

33. Short DB, Trotter KW, Reczek D, et al. (1998) An apical PDZ protein anchors the cystic fibrosis transmembrane conductance regulator to the cytoskeleton. J. Biol. Chem. 273: 19797-19801. 
34. Moyer BD, Denton J, Karlson KH, et al. (1999) A PDZinteracting domain in CFTR is an apical membrane polarization signal. J. Clin. Inv. 104: 1353-1361.

35. Sun F, Hug MJ, Bradbury NA, Frizzell RA. (2000) E3KARP mediates the association of ezrin and protein kinase A with the cystic fibrosis transmembrane conductance regulator in airway cells. J. Biol. Chem. 275: 2953929546.
36. Sun F, Hug MJ, Lewarchik CM, Yun C, Bradbury NA, Frizzell RA. (2000) Protein kinase A associates with cystic fibrosis transmembrane conductance regulator via an interaction with ezrin. J. Biol. Chem. 275: 14360-14366.

37. Rothwarf DM, Karin M. (1999) The NF $\kappa$ B Activation Pathway: A Paradigm in Information Transfer from Membrane to Nucleus. Science's STKE available at: http:// www.stke.org/cgi/content/full/OC_sigtrans;1999/5/rel 